\title{
Performance and perspectives of the diamond based Beam Condition Monitor for beam loss monitoring at CMS.
}

\author{
Moritz Guthoff $^{\star a}$, Wim de Boer ${ }^{b}$, Anne Dabrowski ${ }^{a}$, Florian Kassel ${ }^{a b}$ and David \\ Stickland ${ }^{c}$ \\ ${ }^{a}$ CERN, Geneva, Switzerland \\ ${ }^{b}$ Institut für Experimentelle Kernphysik, KIT, P.O. Box 6980, 76128 Karlsruhe, Germany \\ ${ }^{c}$ Princeton University, Princeton, NJ 08544-0708, USA \\ E-mail: moritz.guthoffecern.ch, wim.de.boer@kit.edu, \\ anne.dabrowski@cern.ch, florian.kassel@kit.edu, \\ david.sticklandecern.ch
}

\begin{abstract}
At CMS, a beam loss monitoring system is operated to protect the silicon detectors from high particle rates, arising from intense beam loss events. As detectors, poly-crystalline CVD diamond sensors are placed around the beam pipe at several locations inside CMS. In case of extremely high detector currents, the LHC beams are automatically extracted from the LHC rings.

Diamond is the detector material of choice due to its radiation hardness. Predictions of the detector lifetime were made based on FLUKA monte-carlo simulations and irradiation test results from the RD42 collaboration, which attested no significant radiation damage over several years. During the LHC operational Run1 (2010 - 2013), the detector efficiencies were monitored. A signal decrease of about 50 times stronger than expectations was observed in the in-situ radiation environment. Electric field deformations due to charge carriers, trapped in radiation induced lattice defects, are responsible for this signal decrease. This so-called polarization effect is rate dependent and results in a non-linearity of the detector response. Measurements using the transient current technique reveal the electric field distribution. Online measurements and laboratory analysis of polarization effects in diamond sensors are presented. Electric field calculations and estimations of TCT pulse shapes show good agreement with measurements.
\end{abstract}

Technology and Instrumentation in Particle Physics 2014,

2-6 June, 2014

Amsterdam, the Netherlands

\footnotetext{
* Speaker.
} 


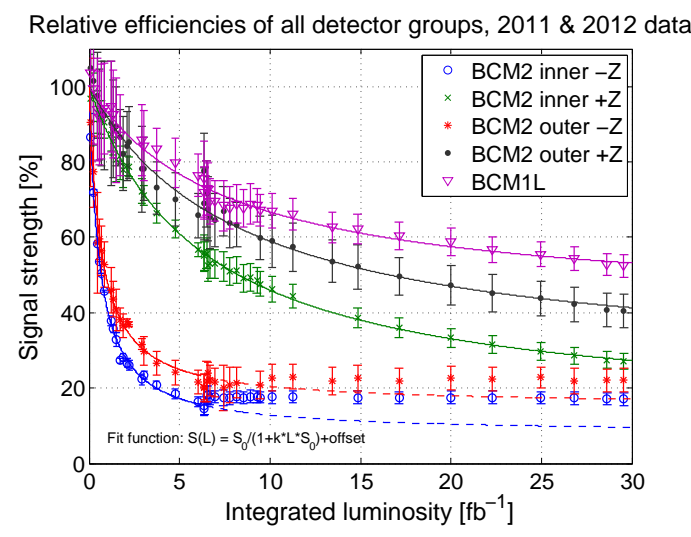

Figure 1: Decrease in detector sensitivity as function of integrated luminosity during Run 1 operation.

\section{Introduction}

The Beam Condition Monitor 2/1L (BCM2/1L) at CMS is used to protect the Si Tracker from catastrophic beam loss events. $1 \times 1 \mathrm{~cm}^{2}$ poly-crystalline CVD diamonds with a thickness of about $400 \mu \mathrm{m}$ are used as detectors. In operating conditions $200 \mathrm{~V}$ bias voltage is applied leading to an average internal field of about $0.5 \mathrm{~V} / \mu \mathrm{m}$. The detector current is measured with the readout electronics that is also used for LHC beam loss monitoring system. In case of extremely high measured signals, which can only arise from potentially damaging beam loss events, the LHC beam are automatically dumped. [1]

During operational Run 1 (2010 - 2013), it was observed that the measured signals are almost entire due to collision product, hence the signal should be proportional to the instantaneous luminosity. In rare events significant signals due to beam loss were observed. [2]

In order to ensure proper operation, the signal output was monitored and compared to the instantaneous luminosity. The signal efficiency at the start of operation was defined as $100 \%$. Figure 1 shows the relative decrease of the detectors, installed at different locations, as function of integrated luminosity. The difference in signal decrease is due to the different radiation environments where the detectors are placed in [3]. The decrease of signal was significantly higher compared to the original expectations [1]. It is likely that the strong decrease is due to charge carriers trapped at radiation damage induced defects, which deform the electric field and hence lower the signal output. The intensity of this field deformation, referred to as polarization, is stronger in a high rate environment, resulting in a strong signal decrease during operation [4].

\section{Transient current technique measurements}

The electric field inside a sensor can be determined by the Transient-Current-Technique (TCT): After introducing electron-hole pairs on one side of the detector by a radioactive $\alpha$ source, one observes the current generated by the drift of the charge carriers due to the electric field in the sensor. This current as function of time is proportional to the electric field as function of distance. By creating the charge carriers either at the cathode or the anode, the drift from electrons or holes can be observed. The used TCT setup consists of a Picosecond 5531 bias tee, a MITEQ AM-1309 am- 


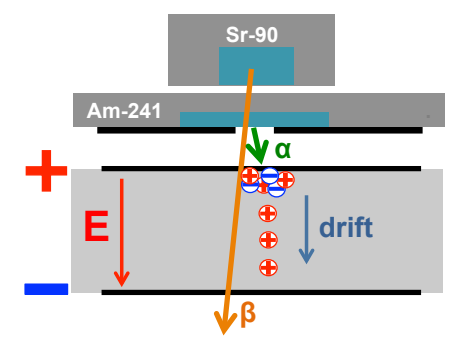

Figure 2: Placement of the sources and particle environment during TCT measurements. The ${ }^{241}$ Am source emits $\alpha$ particles directly on the diamond, which are used for the measurement. The ${ }^{90} \mathrm{Sr}$ source creates MIP-like radiation environment.

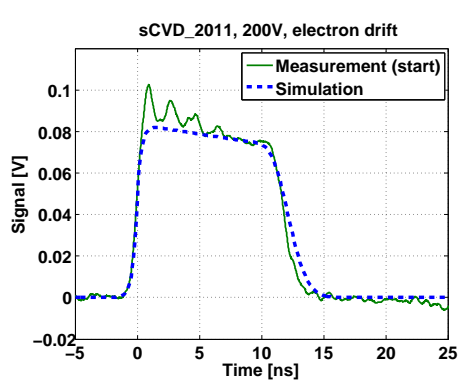

(a) TCT signal with unpolarized diamond

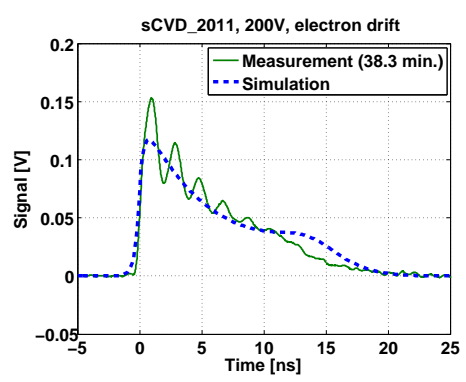

(b) TCT signal for electron drift of polarized diamond

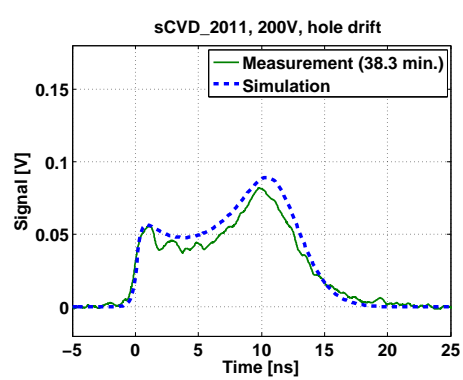

(c) TCT signal for hole drift of polarized diamond

Figure 3: TCT measurements of a single-crystalline CVD diamond. Fig. 3(a) shows the signal shape immediately after the HV was switched on, where no charge carriers are trapped at the lattice defects yet. Fig. 3(b) and 3(c) show the pulse shapes after $\sim 40$ minutes of measurement time, where charge trapped at defects deform the internal electric field and hence the TCT pulse shape.

plifier, a Tektronix TDS 5104B oscilloscope and a Keithley 2410 as HV source. The amplifier has a $50 \mathrm{~dB}$ amplification and a bandwidth of $1 \mathrm{GHz}$, which is the limiting factor in terms of bandwidth for the whole system. As $\alpha$ source a $3.56 \mathrm{kBq}^{241} \mathrm{Am}$ source was used. In addition, a $3.56 \mathrm{MBq}$ ${ }^{90} \mathrm{Sr}$ source was used to pump the detector. During the measurement, both sources were used at the same time (see fig. 2). The $\beta$ particles from the ${ }^{90} \mathrm{Sr}$ source penetrate the thin $\alpha$ source and the diamond. The signals generated by the $\alpha$ particles, which are used to probe the electric field, are significantly higher than the signals of the $\beta$ particles. The trigger threshold of the scope is set to record only signals from the alpha particles. The ${ }^{90} \mathrm{Sr}$ source is used to deposit charge in the whole bulk material, which reflects the radiation environment of the detector in operation.

Since $\alpha$-particles are stopped in any material, an external trigger can't be used. The oscilloscope is set to trigger on the pulses directly. About one pulse is stored by the PC per second. A time dependence of the signal shape is shown by averaging several groups of traces close in time. For the averaging, the curves are realigned using a software discrimination, with a discriminator threshold chosen just above noise level.

The single-crystalline CVD diamond, analyzed with the TCT, was installed in BCM2 during the operation of 2011 and received a $24 \mathrm{GeV}$ proton equivalent fluence of about $5.7 \times 10^{14} \mathrm{~cm}^{-2}$ [4]. It has a thickness of $460 \mu \mathrm{m}$ and the operational $\mathrm{HV}$ of $200 \mathrm{~V}$ was used, giving an average 


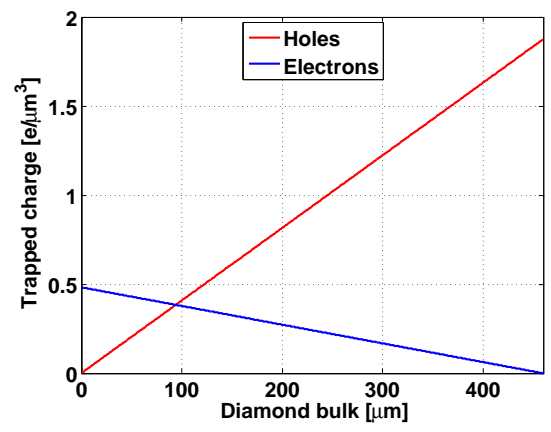

(a) Distribution of trapped space charge

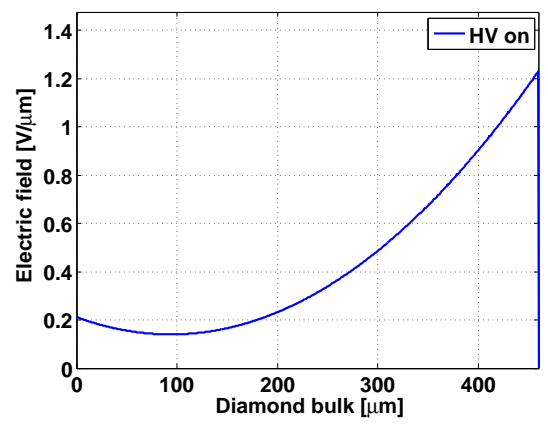

(b) Electric field

Figure 4: Space charge and electric field distribution matching the measured TCT pulses shown in fig. 3(b) and fig. 3(c).

electric field of about $0.43 \mathrm{~V} / \mu \mathrm{m}$. TCT measurements of this detector using only an $\alpha$ source are shown in [3].

At first, the HV is switched off, but the sources are applied to pump the diamond. Any residual polarization field is removed. The data taking starts with the switch-on of the HV. TCT pulses measured immediately at the start, are taken while the electric field is constant in the bulk, as no charge is trapped at the lattice defects yet. The TCT pulse, immediately after the HV was switched on, is shown in fig. 3(a). A square pulse is measured as expected for a constant electric field.

Slowly, charge carriers are trapped and space charge builds up, which leads to a deformation of the electric field and a change in shape of the measured pulses. After $\sim 40$ minutes, the measured pulses are deformed as shown in fig. 3(b) and fig. 3(c). Conclusions on the electric field can be drawn by analyzing the measured pulse shapes, however it is not possible to directly calculate the electric field from the pulse shapes due to non linear transport characteristics of the charge carriers. Thus the approach was chosen to find an electric field distribution, which would create such TCT pulses.

\section{Simulation of electric field}

The drift behavior of the charge carriers depends on the electric field itself, hence the measured TCT signals are not linearly correlated with the electric field. In order to understand the electric field distribution a 1-D simulation of the electric field was performed. As input parameters only the relative strength of the trapping and de-trapping for electrons and holes is given. The trapping probability is evenly distributed inside the bulk, since it is expected, that the amount radiation induced defects is the same everywhere in the diamond. The charge carrier density is assumed to increase linearly towards the anode for electrons and towards the cathode for holes. The trapping parameters and the charge carrier density is used to calculate the trapped space charge. For a given space charge distribution, the electric field is calculated. Since the applied HV is constant, the integral over the electric field is also constant. The reduction in electric field due to polarization is compensated by the HV supply by further charging up the electrodes. The amount of charge on 
the electrodes is calculated and included in the electric field calculation, resulting in an unchanged average electric field.

For a given electric field, a TCT pulse can be estimated by calculating the drift of the charge carriers, taking into account a small amount of charge trapping and longitudinal diffusion. The charge drift is converted to a voltage value, using the electronic specification of the used measurement setup. The hereby calculated pulse shape can be compared with the measured pulse.

The TCT pulse of an unpolarized detector is estimated by assuming no trapped space charge and hence a constant electric field corresponding to the externally applied field. The simulated TCT curves matches well the measured curve (fig. 3(a)).

The TCT pulses in fig. 3(b) and fig. 3(c) are shown together with an simulation of the TCT pulses. The space charge and the electric field of this simulation are shown in fig. 4. The electric field is increase towards the cathode since a strong trapping of holes is given.

\section{Conclusions}

With TCT measurements it is possible to show that an irradiated SCVD diamond detector builds up space charge, resulting in an deformed electric field. By applying a hot $\beta$ source, the diamond was placed in a radiation environment similar to operational conditions, while at the same time performing TCT measurements with a weak $\alpha$ source. Electric field calculations of an assumed space charge distribution are used to estimate the shape of the TCT pulse, where an agreement was achieved by assuming hole trapping being dominant over electron trapping.

\section{Acknowledgments}

We thank the following people or groups for their support: Robert Eber, Andreas Nürnberg and Pia Steck from the IEKP at KIT Karlsruhe, the CMS group of DESY Zeuthen, CERNs BE/BI group, Eleni Berderman from GSI, Germany and the RD42 collaboration.

\section{References}

[1] S. Müller, The Beam Condition Monitor 2 and the Radiation Environment of the CMS Detector at the LHC, Ph.D. thesis, IEKP-KA/2011-01, Universität Karlsruhe, CERN-THESIS-2011-085, 2011.

[2] M. Guthoff, Design and Experiences with the Beam Condition Monitor as Protection System in the CMS Experiment of the LHC, proceedings of European Workshop on Beam Diagnostics and Instrumentation for Particle Accelerators, DIPAC'11, Hamburg, Germany, 2011.

[3] M. Guthoff et. al., Radiation damage in the diamond based beam condition monitors of the CMS experiment at the Large Hadron Collider (LHC) at CERN, NIM A 730 (2013) 168-173, DOI:10.1016/j.nima.2013.05.041.

[4] M. Guthoff, Radiation Damage to the diamond-based Beam Condition Monitor of the CMS Detector at the LHC, Ph.D. thesis, KIT Karlsruhe, IEKP-KA/2014-01, 2014. 